\title{
PSF reconstruction via full turbulence characterization and end-to-end simulations
}

\author{
Clémentine Béchet ${ }^{\mathrm{a}}$, Boris Ayancán ${ }^{\mathrm{a}}$, Andrés Guesalaga $^{\mathrm{a}}$, Carlos Correia $^{\mathrm{b}}$, Benoit Neichel ${ }^{\mathrm{b}}$, \\ Elena Masciadri ${ }^{\mathrm{c}}$, and Rodolphe Conan ${ }^{\mathrm{d}}$ \\ ${ }^{a}$ Engineering School, Pontificia Universidad Católica de Chile, Santiago, Chile \\ ${ }^{\mathrm{b}}$ Laboratoire d'Astrophysique de Marseille, Marseille, France \\ ${ }^{\mathrm{C} I N A F}$ - Arcetri Astrophyisical Observatory, Florence, Italy \\ ${ }^{\mathrm{d}}$ Giant Magellan Telescope Corporation, Pasadena, USA
}

\begin{abstract}
Enhancement and wise archiving of astronomical images require an accurate estimate of the observational Point Spread Function (PSF). Although modelling of the telescope and its optics is a well-understood problem, PSF reconstruction becomes challenging when the observations include adaptive optics $(\mathrm{AO})$ correction. The approach presented in this paper consists in feeding an end-to-end (E2E) simulation of the telescope, the instrument and its environment with the characterized disturbances from the telemetry and AO loop data, in order to produce the estimated PSFs. This method benefits from the developments made in the last years with respect to the estimation of external disturbances during $\mathrm{AO}$ correction, such as turbulence profile and its dynamics as well as sensor noise and vibrations characteristics. In particular, characterization of the turbulence profile in terms of strength, $C_{n}^{2}(h)$, and outer scale, $L_{0}(h)$, is considered with an example on on-sky recorded AO telemetry from the GALACSI AO system. Once identified, the internal and external parameters of the observing conditions are used as inputs to carry out E2E simulations of the optical propagation and estimate the PSF. The method can be regarded as a "brute force" approach, as it is highly intensive in computer power; particularly for the ELTs. However, its ability to integrate complex combination of effects from all disturbances and not relying on analytical approximations for the aliasing or fitting errors makes the approach worth of a deeper study. E2E simulations have been used before in PSF reconstruction, but limited to a theoretical modelling of the system. Here, the development of the E2E simulation part is an ongoing work. A simplified AO system similar to the GALACSI WFM is currently simulated to obtain the PSF estimates and illustrate how such approach allows to account for the anisoplanatic effects and for the influence of the outer scale values.
\end{abstract}

Keywords: point spread function, reconstruction, turbulence profiling, end-to-end simulations

\section{INTRODUCTION}

The scientific programs of the Giant Segmented Mirror Telescopes (GSMTs) rely on ambitious goals of precision and accuracy in terms of photometry and astrometry for the observations of crowded fields, for exoplanets research, for orbits measurements near the Galactic Center, for graviational lensing or also to study the dynamics of galaxies. ${ }^{1-4}$ To meet such goals, the knowledge of the instrumental response during the observations, the socalled point spread function (PSF), is essential. In other words, astronomical data should be archived together with their corresponding PSF to achieve the best possible post-processing and analysis of the data.

Although modelling of the telescope and its optics is a well-understood problem, the estimation of the PSF becomes challenging when the observations include adaptive optics $(\mathrm{AO})$ correction. PSF reconstruction or PSF estimation methods for AO-corrected observations has been studied over the last 20 years, starting with the work of J.P. Véran et al. ${ }^{5}$ for on-axis classical AO. The research field became more and more complex to include effects of crucial importance when new technologies such as laser based and wide-field AO systems were introduced (see

Further author information: (Send correspondence to Clémentine Béchet)

Clémentine Béchet: E-mail: cbechetp@ing.puc.cl 
for instance the work of Fusco et al. ${ }^{6}$ for anisoplanatism and the one of Gilles et al. ${ }^{7}$ for tomography). The rare on-sky demonstrations of PSF reconstruction is a clear sign of the complexity of the whole process. ${ }^{8-11}$

Providing reliable estimates of the PSF for AO-corrected observations is still a major challenge, mainly due to the wide-range of parameters having an influence on it. Knowledge of the instrument (AO system \& telescope) and atmospheric parameters is mandatory. First, accurate estimation of the atmospheric parameters (seeing, vertical profile, wind speed and its impact on temporal AO errors) is critical. Furthermore, parameters of the telescope, of the AO control, of its configuration and of the optical design are also key. It is hard to ensure that a model-based approach could properly reflect the combination of effects induced by all these parameters, some of them combining non-linearly. Hence, in this paper, the authors present a PSF estimation strategy based on end-to-end simulations of the AO system, fed by input parameters that are obtained during a pre-process stage of full characterization of the system (telescope \& $\mathrm{AO}$ ) and its atmospheric environment.

In Sect. 2, we describe the components and structure of this two-step process for PSF estimation. In Sect. 3, we focus on the recent advances for accurate estimation of the atmospheric profiles involved in the characterization step of the process. In Sect. 4, we illustrate the advances of the project in the step 2 of the PSF estimation process, showing the impact of the outer scale values on the PSFs obtained via simulations.

\section{DESCRIPTION OF THE TWO-STEP PSF ESTIMATION PROCESS}

The two-step process presented in this paper to get estimates of the PSF is summarized in the diagram of Fig. 1. The left part of the diagram details the relevant inputs of this process. We consider three types of inputs:

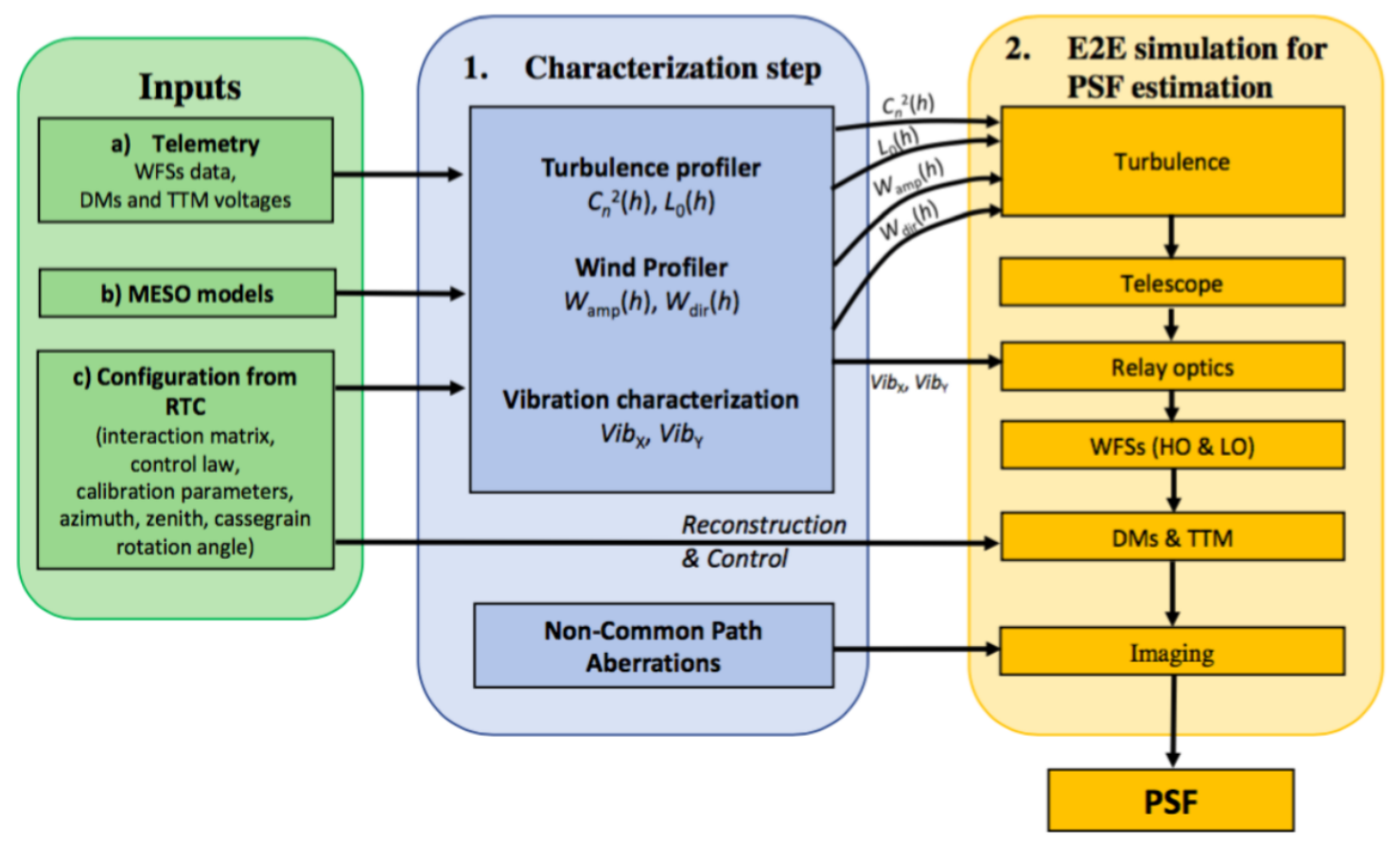

Figure 1. Diagram summarizing the two-step approach for PSF estimation. Step 1: Full characterization of the environmental variables. Step 2: PSF estimation using end-to-end simulations.

- AO telemetry data, i.e. recorded sequences of wavefront sensors (WFS) data and recorded sequences of deformable and tip-tilt mirrors commands;

- the Meso-Nh mesoscale model ${ }^{12}$ together with the Astro-Meso-Nh package for the optical turbulence ${ }^{13}$ and available data associated to the time of the observation; 
- configuration parameters used for AO real-time computations, such as interaction and control matrices, control law, as well as any relevant calibration parameters (e.g. dead actuators, telescope orientation angles).

These data allow to keep in memory the AO scenario and configuration at the time of the observations. They are used as inputs in Fig. 1 for both the step of characterization of the observing conditions (blue column in the middle) and the step of PSF estimation (yellow column on the right).

The step 1 of the process, i.e the characterization step at the center of the diagram of Fig. 1, aims at fully determining the observing conditions and in particular:

- the atmospheric turbulence profile and its evolution during the observation, i.e. the turbulence strength profile $C_{n}^{2}(h)$ and the profile of the outer scale of the turbulence $L_{0}(h)$ depending on the altitude $h$;

- the profile of the wind amplitude, $W_{\mathrm{amp}}(h)$, and of its direction, $W_{\mathrm{dir}}(h) ;^{14}$

- the temporal or frequency characteristics of possible 2D-vibrations, Vib.

This characterization process benefits from the developments made in the last years with respect to the estimation of external disturbances during AO correction. In addition, the characterization step is expected to be completed with the information on the existing non-common path aberrations, as mentioned in Fig. 1.

Once the system and the conditions are fully characterized, the identified parameters are used to configure the conditions to be simulated in Step 2 (see the right part of diagram in Fig. 1) and obtain estimates of the PSF. The characteristic parameters of the atmospheric profiles are used to generate adequate random draws of the atmosphere for the simulations. The AO system is fully simulated as well as the science path in order to deduce long-exposure PSF estimates. Thanks to this approach, the PSF accounts for the anisoplanatism effects, as simplest approaches also do ${ }^{15}$ but accounts also for many other contributions of the AO system dynamics and control loop ${ }^{7,11,16}$ to the residuals phase distortions. It can in addition allow to combine non-linear effects without the possible limitations of an analytical model-based approach.

Our project aims at having all the elements of the diagram implemented and integrated in a dedicated code able to provide estimates of the PSF at the scale of an ELT in a reasonable computational time. In this paper, we present the advances made in step 1 and step 2 so far. For step 1, in Sect. 3, we focus on the recent advances made to obtain an accurate profiling of both the turbulence strength profile $\left(C_{n}^{2}(h)\right)$ and the outer scale profile $\left(L_{0}(h)\right)$. For step 2, in Sect. 4, the current status of the implementation of the simulator for PSFs is illustrated by showing the impact of the outer scale values on the PSFs for a laser-based ground-layer AO system correcting in the visible.

\section{ADVANCES IN STEP 1: PROFILING THE OUTER SCALE OF TURBULENCE}

We present in this section the most recent advances made for the characterization step, which are related to the upgrade by Guesalaga et al. ${ }^{17}$ of the SLODAR-type (SLOpe Detection And Ranging) turbulence profiler in order to estimate non-uniform profiles of the outer scale of the turbulence, $L_{0}(h)$. This SLODAR profiler was originally developed for GeMS (Gemini South Multiconjugate AO system) ${ }^{18}$ and the upgraded version is now currently tested on the Adaptive Optics Facility (AOF) at the Very Large Telescope (VLT). The estimation of the outer scale profile is expected to improve the accuracy of the turbulence strength estimation $\left(C_{n}^{2}(h)\right)$ at the same time, allowing for a better fit of the cross-correlations of the WFSs measurements in the SLODAR approach. The knowledge of the variations of the outer scale of the turbulence with the altitude is important to correctly model the anisoplanatism effects on PSF when the low-order reference stars are chosen in a wide field-of-view.

The results presented here were obtained from AO telemetry data recorded on-sky by ESO with the AOF GALACSI wide-field-mode AO system, dedicated to the 3D-Multi-Unit Spectroscopic Explorer (MUSE) instrument. The knowledge of the AO-corrected PSF for this spectrograph is key to distinguish the various observed astrophysical targets in a given field. An accurate turbulence profiler is thus important for this AO-assisted 
instrument. MUSE can benefit either from ground-layer AO correction in wide-field mode (WFM) or from Laser Tomography AO correction in the narrow-field mode (NFM). The AO module providing these corrections is called GALACSI and works with an asterism of 4 lasers guide stars and an additional tip-tilt star located further off-axis in the technical field of view. The ground-layer AO correction of GALACSI WFM aims at providing a seeing enhancement by a factor 2 at $750 \mathrm{~nm}$. We refer the reader to La Penna et al. ${ }^{19}$ for a complete description of GALACSI AO system.

The profiler has been applied on 99 sets of on-sky recorded data with GALACSI WFM. Absolute values $\left(C_{n}^{2}\right.$ and total $\left.r_{0}\right)$ have been calibrated using data from the tests previously made with the ASSIST bench of the AOF, ${ }^{19}$ with 3 phase screens of known turbulence strength placed at known altitudes, namely with phase screens of $r_{0}$ values $12 \mathrm{~cm}, 26 \mathrm{~cm}$ and $26 \mathrm{~cm}$ located at the ground, at $\sim 5.7 \mathrm{~km}$ and at $\sim 8.6 \mathrm{~km}$ respectively. Figure 2 shows the fitting results of the theoretical cross-correlations response functions to the measured WFSs spatial cross-correlations for one of the 99 recorded open-loop AO telemetry sequence provided by ESO (file 2017-05017T0909_HK_0.0_0_100_500_4.fits).

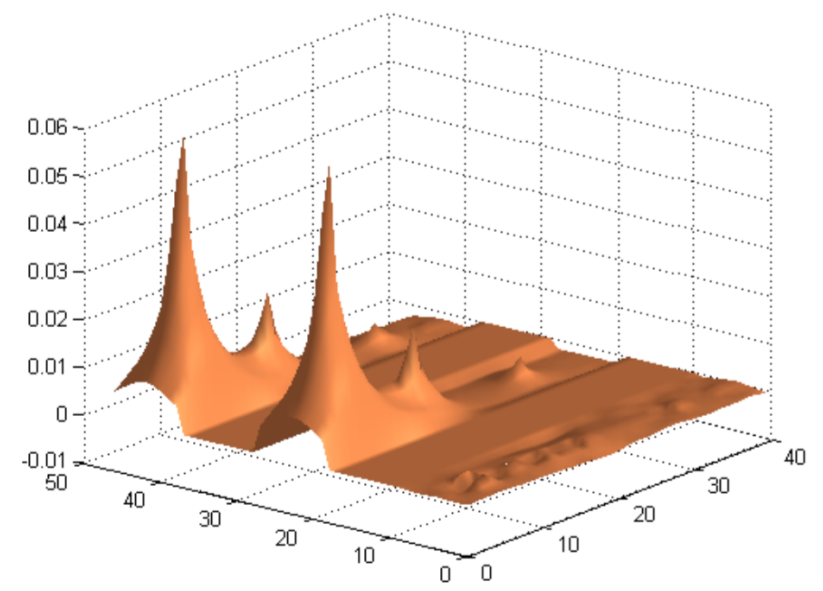

Figure 2. Fitting of the WFSs cross-correlations (top-left strip) to theoretical cross-correlations (central strip). The bottom-right strip is the difference between the two correlations. Axis are set in arbitrary units. We refer to Guesalaga et $a l .{ }^{17}$ for a detailed description of the fitting procedure for the outer scale depending on the altitude.

From this fit of the WFSs cross-correlations, a profile of the turbulence strength $C_{n}^{2}(h)$ and a profile of the outer scale $L_{0}(h)$ have been obtained for this file. They are represented in Fig. 3 (left plot for $C_{n}^{2}$ and right plot for $\left.L_{0}\right)$ as a function of the height along the line of sight. The information about the zenith angle of for these recorded data was missing, so the corresponding altitude is not known.

This set of recorded data has an interesting feature which is a very different outer scale length, $L_{0}$, fitted for the upmost layer (at $7.8 \mathrm{~km}$ ) compared to the other ones. The low layers have $L_{0}$ value between 5 and 22 meters, which are usual numbers found with slope detection and ranging (SLODAR) profilers from AO telemetry on 8 -meter telescopes ${ }^{20}$ However, the very different value $L_{0}=65 \mathrm{~m}$ found for the outer scale of the upmost layer is quite unusual. This may be explained by the fact that this layer has low energy and the signal-to-noise ratio of the fit of the width of the associated cross-correlation peak (the lowest peak visible in the first strip of Fig. 2) is very low.

Nevertheless, this peculiar situation of a very large outer scale for the upmost layer compared to the other ones is of great interest to study the importance of determining the outer scale profile for the PSF estimation. This is true because in addition for the considered ground-layer AO in the visible the tip-tilt reference may be located far enough off-axis such that the anisoplanatism effect associated to a peculiar $L_{0}$ value can have significant impact on the PSF correction quality. 

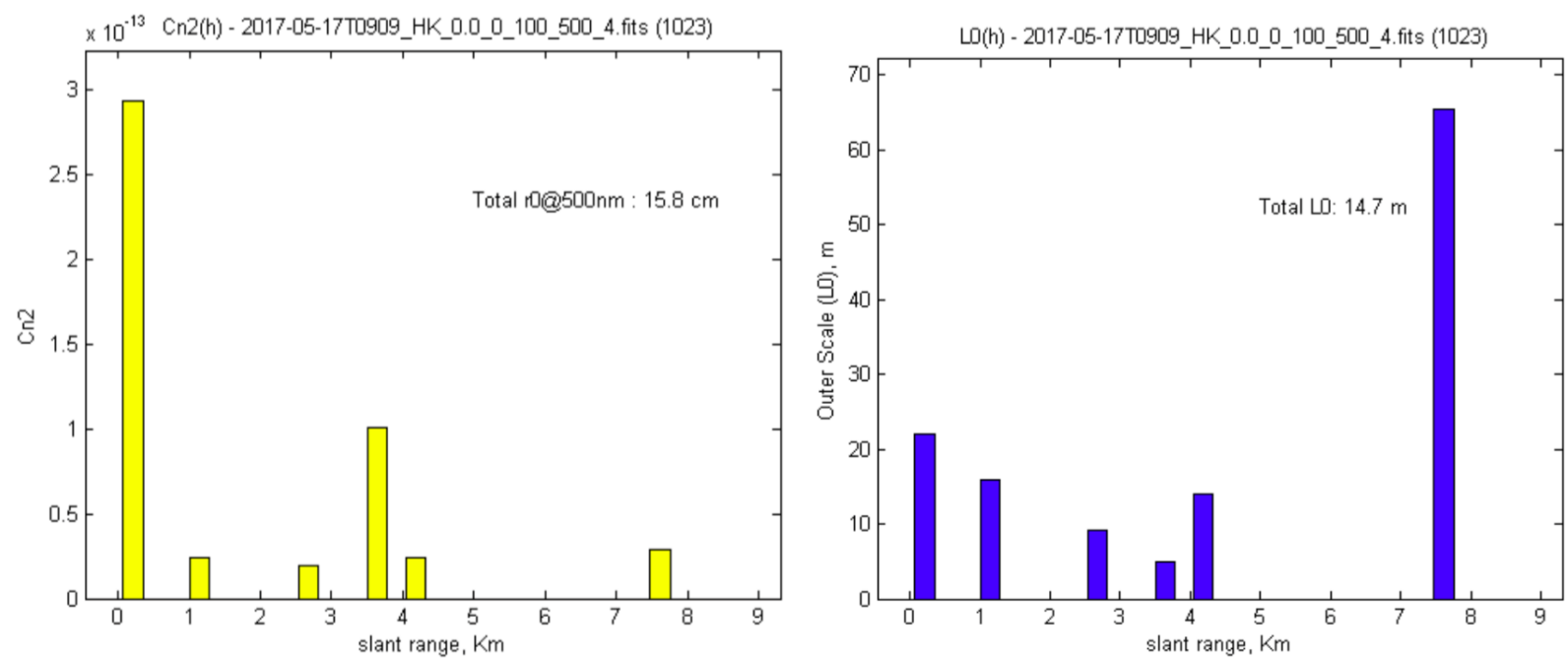

Figure 3. Estimated profiles of turbulence strength, $C_{n}^{2}$, and outer scale of turbulence, $L_{0}$, as a function of the height along the line of sight.

\section{ADVANCES IN STEP 2: ANISOPLANATISM AND OUTER SCALE EFFECTS ON ESTIMATED PSF VIA SIMULATIONS}

The second part of the two-step PSF estimation process presented in this paper consists in running E2E simulations reproducing the observing conditions and allowing to estimate the long-exposure AO-corrected PSFs. So far, only parts of the components of this step 2 are integrated to the code, namely:

- a simulator of 3D turbulent atmosphere with modal static sensing and correction in phase space;

- a simulator of residual PSFs in a set of targets directions as outputs.

Together with the turbulence profiler presented in Sect. 3, it allows to study the anisoplanatism effects and the profilers accuracy influence on the PSF estimates using simulations according to the scheme shown in Fig. 4.

In the right column of Fig. 4, the turbulent atmosphere generation and the simulated AO system configuration are obtained using the object-oriented Matlab adaptive optics package (OOMAO). ${ }^{21}$ The simulated wavefront sensing directly measures the phase without noise. The tip-tilt (TT) is removed from the phase measurements in the directions of the high-order (HO) reference stars. The phase is corrected on the Zernike modal basis up to a given order $N$ ( $N=500$ in the following). We do not simulate any non-common path aberrations neither vibrations so far. In addition, the simulation applies a static and non-delayed correction, which means that there is no servo loop control implemented so far. Some parts of the end-to-end simulation process are implemented using powerful GPUs to reduce the processing time to a couple of orders of magnitude with respect to the realtime case. Later, when the complete end-to-end simulations are implemented, the authors will analyze how the real-time gap can be reduced using dedicated hardware modules (e.g. FPGAs) for the most stable parts of the code such as turbulence generation, propagation and WFS simulations.

As already mentioned in Sect. 3, it is interesting to study an AO configuration like GALACSI WFM when the TT star is located far off-axis from the instrument field-of-view and with AO-correction in the visible in order to highlight the anisoplanatism and the outer scale effects on the PSF estimates. The chosen configuration to be simulated in this section is thus summarized in Table 1 and is based on ASSIST AOF test bench configurations provided by ESO. It corresponds to an AO configuration similar to GALACSI WFM conditions with the geometry shown in Fig. 5. The step 2 of the process allows us to simulate long-exposure PSFs for this configuration and deduce a map of the Ensquared energy (EE) in $0.2 " \times 0.2$ " areas over the instrument $64 " \times 64$ " field-of-view. This map is shown on the left plot of Fig. 6 with the EE expressed in arbitrary units. The right plot of Fig. 6 shows the gain in EE obtained in these conditions, when AO correction is applied compared to no AO correction. 


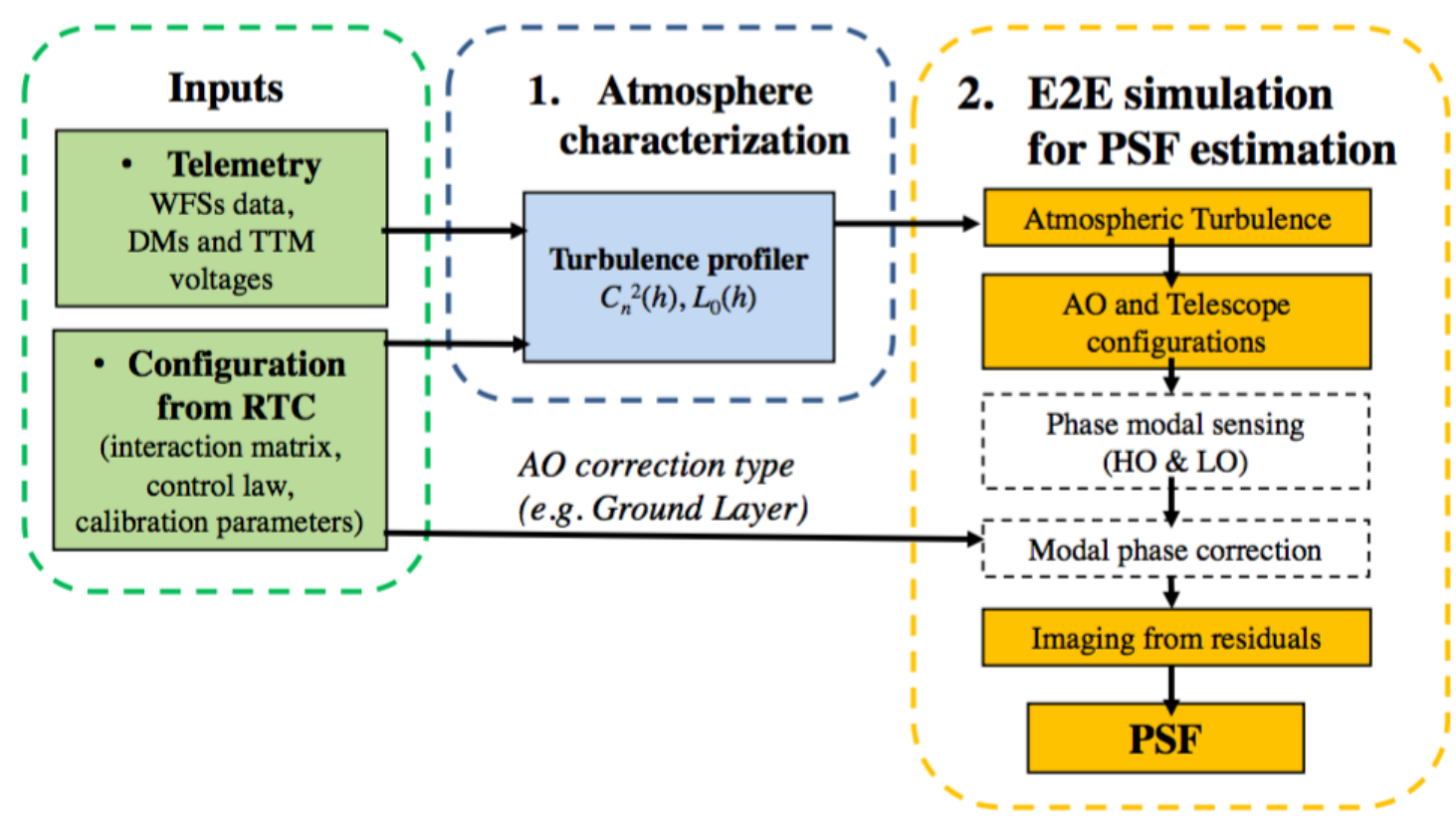

Figure 4. Diagram summarizing the implemented parts of the process so far. These elements are used for the study of the outer scale impact on PSF in the rest of the paper.

Table 1. Parameters of simulations for PSF estimation (Step 2).

\begin{tabular}{|c|c|c|}
\hline TELESCOPE & Diameter $(\mathrm{m})$ & 8 \\
\hline \multirow{4}{*}{$\begin{array}{c}\text { SOURCES } \\
4 \text { HO guide stars } \\
1 \text { TT guide star } \\
\text { 64 PSF stars }\end{array}$} & HO Stars position & 64 " off-axis \\
\cline { 2 - 3 } & TT star position & $120 "$ off-axis \\
\cline { 2 - 3 } & PSF stars grid & $8 \times 8$ \\
\cline { 2 - 3 } & FOV of stars grid & $64 " \times 64 "$ \\
\cline { 2 - 3 } & All sources heights & Infinity \\
\hline \multirow{3}{*}{$\begin{array}{c}\text { ATMOSPHERE } \\
\text {. } 3 \text { layers }\end{array}$} & $r_{0}(\mathrm{~m})$ at $500 \mathrm{~nm}$ & 750 \\
\cline { 2 - 3 }$($ ASSIST config.) & $L_{0}(\mathrm{~m})$ & 0.15 \\
\cline { 2 - 3 } & fractions of $C_{n}^{2}$ & {$[0.66,0.17,0.17]$} \\
\cline { 2 - 3 } & heights $(\mathrm{m})$ & {$[200,5700,8600]$} \\
\cline { 2 - 3 } & wind directions $(\mathrm{rad})$ & {$[\pi, 0, \pi / 2]$} \\
\cline { 2 - 3 } & wind speeds $(\mathrm{m} / \mathrm{s})$ & {$[8,16,12]$} \\
\hline \multirow{3}{*}{$\begin{array}{c}\text { LONG-EXPOSURES } \\
\text { for PSF estimation }\end{array}$} & Single frame exposure & instantaneous \\
\cline { 2 - 3 } & Time btw frames $(\mathrm{ms})$ & 50 \\
\cline { 2 - 3 } & Total time exposure $(\mathrm{s})$ & 100 \\
\cline { 2 - 3 } & Accumulated frames & 2000 \\
\hline
\end{tabular}

If one assumes that there is the same amount of energy in the atmospheric turbulence but that the outer scale value $L_{0}$ is different from $25 \mathrm{~m}$, the estimated PSFs without AO correction does not change. This has been checked using the simulations process in Step 2, keeping constant the $C_{n}^{2}$ distribution but modifying the $r_{0}$ value depending on $L_{0}$ value. It has been done for $L_{0}=8,25$ and $200 \mathrm{~m}$, and the obtained maps of EE without AO correction were the same. When $L_{0}$ increases, the $\mathrm{AO}$ correction allows to achieve an increased gain in EE due to lower anisoplanatism between the directions of the AO-corrected PSF stars and the direction of the TT star.

This is effectively illustrated by the maps of gain in EE we have obtained in Fig. 7. On the left plot, with a simulated $L_{0}=8 \mathrm{~m}$, the gain in EE is slightly degraded compared to the case of $L_{0}=25 \mathrm{~m}$ in Fig. 6 . Despite 


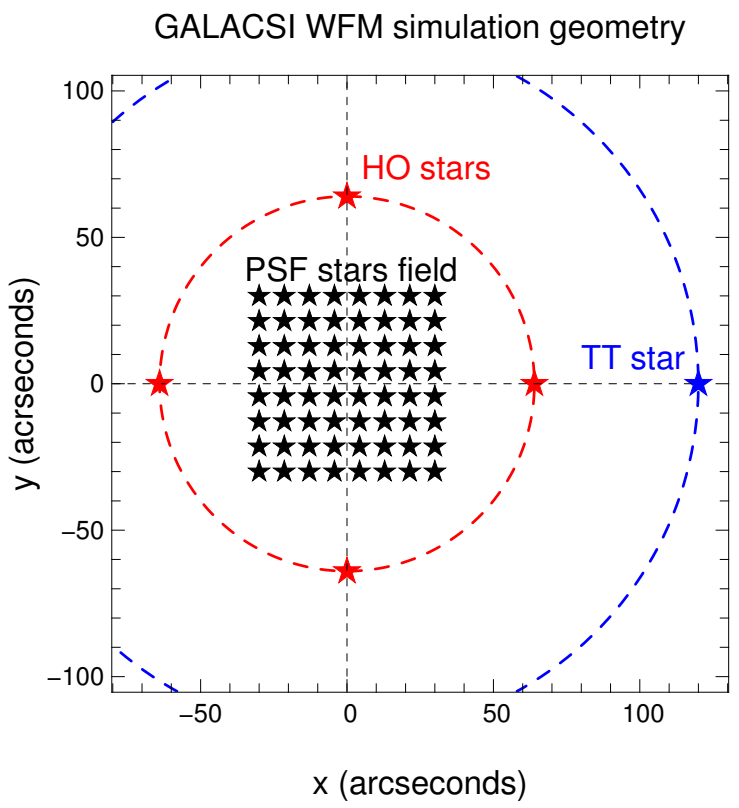

Figure 5. Geometry of the simulated configuration for GALACSI WFM estimation of PSFs. The high-order (HO) guide stars asterism is shown in red, the tip-tilt (TT) guide star in blue and the field of estimated PSFs is covered by an $8 \times 8$ square grid of stars. See Table 1 for more details.

Energy on $0.2^{\prime \prime} \times 0.2^{\prime \prime}$ square across the FoV. LO= $25 \mathrm{~m}$

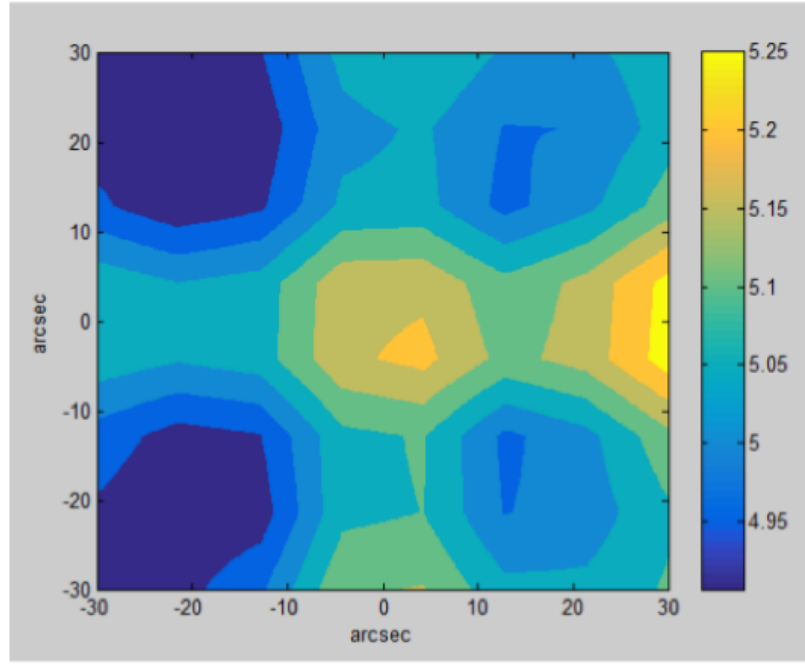

Ensquared Energy distribution after AO correction, arbitrary units

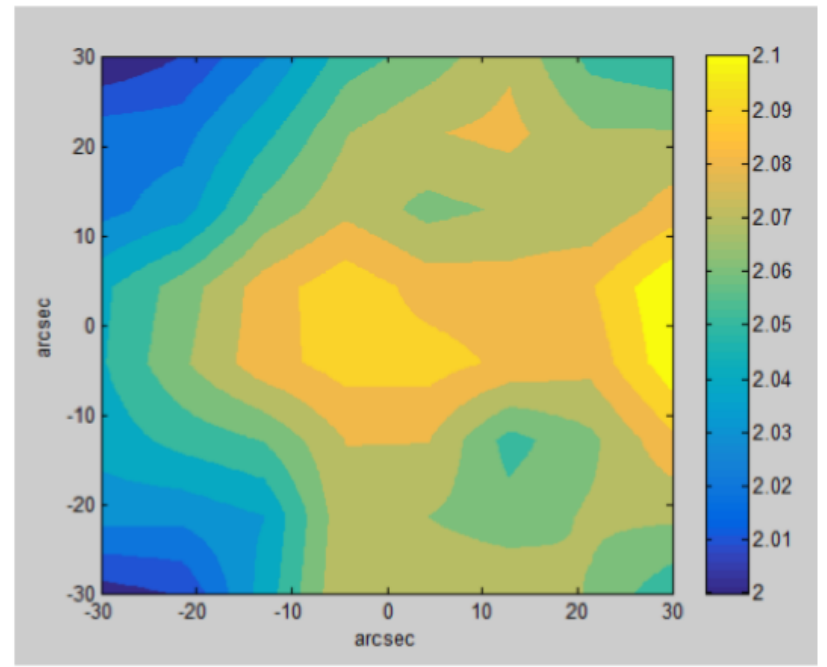

Gain of Ensquared Energy with $\mathrm{AO}$ corrections, relative to $\mathrm{AO}$ off

Figure 6. Left: Map of EE values (arbitrary units) in 0.2 " $\times 0.2$ " areas, covering the 64 " $\times 64$ " corrected FOV of the instrument. The simulated atmosphere has a constant outer scale in altitude, $L_{0}=25 \mathrm{~m}$. Right: Map of the gain in EE obtained for this simulated atmosphere between AO-corrected PSFs and PSFs with no AO.

a lower gain in $\mathrm{EE}$, the values are slightly more uniform across the field. On the contrary, when $L_{0}=200 \mathrm{~m}$ (right plot of Fig. 7), the gain in EE is much less uniform across the field (from 2.25 to 2.5), but it is significantly improved. 


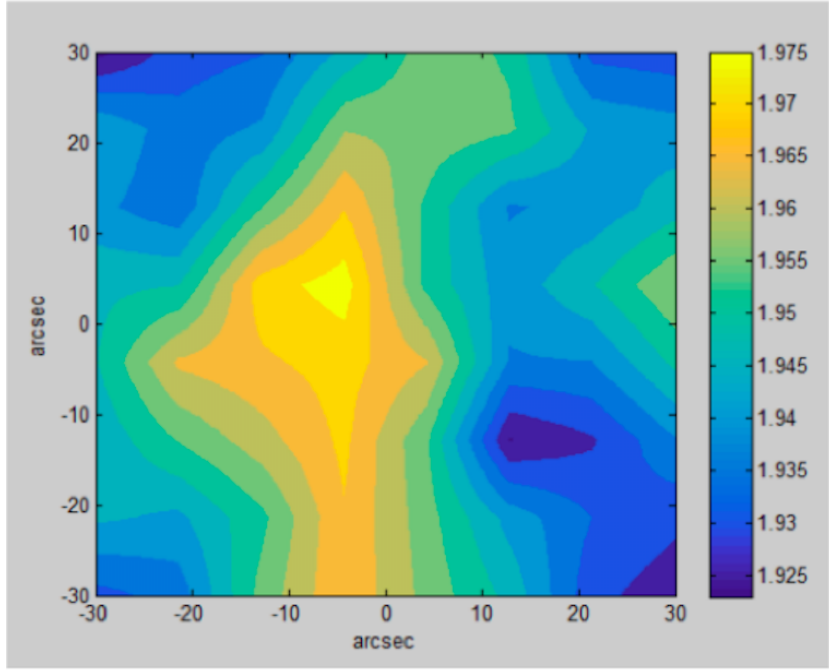

Gain for $\mathrm{L} 0=8 \mathrm{~m}$

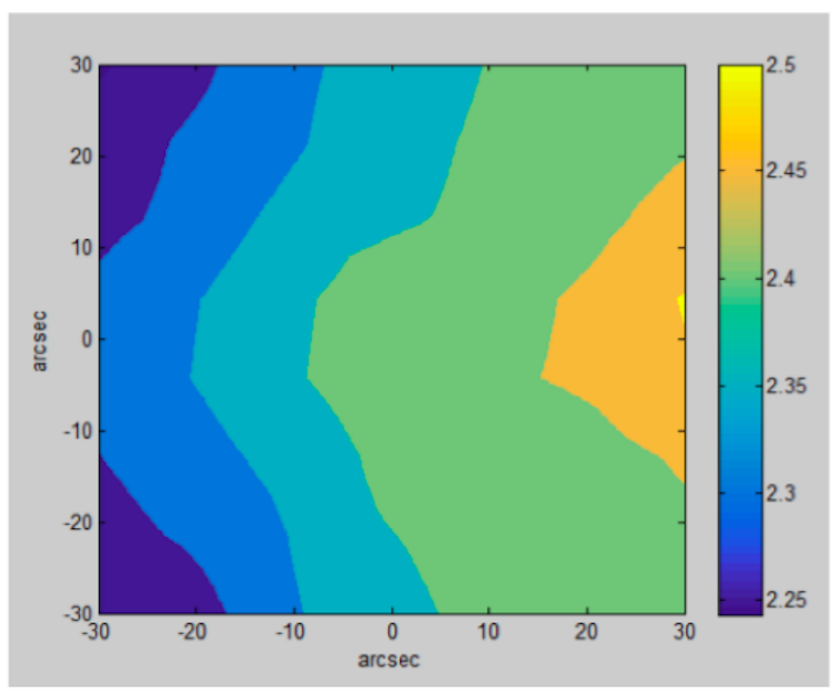

Gain for L0=200m

Figure 7. Comparison of maps of gain in EE when AO-correction is used compared to no AO correction for a given simulated atmosphere with constant outer scale value $L_{0}$ over the altitude. EE has been obtained for each case (each $L_{0}$, with AO-correction and without AO-correction) estimating the PSF from simulations (step 2). Left: $L_{0}=8 \mathrm{~m}$. Right: $L_{0}=200 \mathrm{~m}$.

\section{CONCLUSIONS AND FURTHER WORK}

A two-step process is presented for the PSF estimation of AO-corrected observations. The first stage aims at fully characterizing the observing conditions (atmosphere and telescope) and the estimated parameters are next used to feed an AO simulator in order to estimate the long-exposure PSFs. The paper shows preliminary results using the currently implemented blocks of this PSF estimation process.

The characterization of the atmospheric conditions benefits from the recent advances in SLODAR profiling techniques allowing to estimate a variable outer scale of turbulence depending on the altitude. Such profiling has been applied on AO telemetry data provided by ESO and recorded on the GALACSI WFM AO system, and it effectively evidences fluctuations of the estimated outer scale. The estimation of the outer scale and its dependence on height are important for PSF estimation. In the second step of the process, we highlight the impact of the outer scale value on the PSF shape through variations of the gain in ensquare energy for a wide-field AO system where the low-order star is located far off-axis with respect to the corrected field of view.

Further work is required to be able to conclude on the importance of the outer scale profiling for the accuracy of the PSF estimation. The results of turbulence profiling on the GALACSI WFM will be analyzed further with both simulations and other on-sky recorded data, comparing the estimated profiles of $C_{n}^{2}(h)$ and $r_{0}$ depending on whether the outer scale is forced to be constant or not with the layer heights. This will allow to understand how important the outer scale profiling is for the PSF reconstruction.

The project will also include further development of the E2E simulation code, including the AO control contribution to the PSF estimates. Finally, the most computationally demading parts of these E2E simulations will be accelerated with the use of dedicated hardware.

\section{ACKNOWLEDGMENTS}

A. Guesalaga thanks Conicyt (grant Fondecyt-1160236) for supporting this work. 


\section{REFERENCES}

[1] Cameron, P. B., Britton, M. C., and Kulkarni, S. R., "Precision astrometry with adaptive optics," The Astronomical Journal 137(1), 83 (2009).

[2] Schoedel, R., "Accurate photometry with adaptive optics in the presence of anisoplanatic effects with a sparsely sampled psf - the galactic center as an example of a challenging target for accurate ao photometry," Astron. \& Astroph. 509, A58 (2010).

[3] Ammons, A. M., Bendek, E. A., and Guyon, O., "Microarcsecond relative astrometry from the ground with a diffractive pupil," in [Techniques and Instrumentation for Detection of Exoplanets V], Proc.SPIE 8151, $8151-8151-9$ (2011).

[4] Davies, R., Engel, H., Hicks, E., Foerster Schreiber, N. M., Genzel, R., Tacconi, L. J., Eisenhauer, F., and Rabien, S., "Dissecting galaxies with adaptive optics," in [Adaptive Optics Systems II], Proc.SPIE 7736, $7736-7736-9(2010)$.

[5] Véran, J.-P., Rigaut, F., Maitre, H., and Rouan, D., "Estimation of the adaptive optics long-exposure point-spread function using control loop data," J. Opt. Soc. Am. A 14, 3057-3069 (Nov 1997).

[6] Fusco, T., Conan, J.-M., Mugnier, L. M., Michau, V., and Rousset, G., "Characterization of adaptive optics point spread function for anisoplanatic imaging. Application to stellar field deconvolution," Astronomy and Astrophysics Supplement Series 142, 149-156 (Feb. 2000).

[7] Gilles, L., Correia, C., Véran, J.-P., Wang, L., and Ellerbroek, B., "Simulation model based approach for long exposure atmospheric point spread function reconstruction for laser guide star multiconjugate adaptive optics," Appl. Opt. 51, 7443-7458 (Nov 2012).

[8] Jolissaint, L., Neyman, C., Christou, J. C., Wizinowich, P. L., and Mugnier, L., "First successful adaptive optics psf reconstruction at w. m. keck observatory," in [Proceedings of the 2nd AO4ELT conference], (2012).

[9] Ragland, S., Jolissaint, L., Wizinowich, P., and Neyman, C., "Status of point spread function determination for keck adaptive optics," in [Adaptive Optics Systems IV], Proc.SPIE 9148, 9148 - 9148 - 11 (2014).

[10] Ragland, S., Jolissaint, L., Wizinowich, P., van Dam, M. A., Mugnier, L., Bouxin, A., Chock, J., Kwok, S., Mader, J., Witzel, G., Do, T., Fitzgerald, M., Ghez, A., Lu, J., Martinez, G., Morris, M. R., and Sitarski, B., "Point spread function determination for keck adaptive optics," in [Adaptive Optics Systems V], Proc.SPIE 9909, 9909 - 9909 - 18 (2016).

[11] Beltramo-Martin, O., Correia, C., Neichel, B., and Fusco, T., "Point spread function reconstruction for tomographic adaptive optics systems," in [this conference], (2017).

[12] Lafore, J. P., Stein, J., Asencio, N., Bougeault, P., Ducrocq, V., Duron, J., Fischer, C., Héreil, P., Mascart, P., Masson, V., Pinty, J. P., Redelsperger, J. L., Richard, E., and Vilà-Guerau de Arellano, J., "The meso-nh atmospheric simulation system. part i: adiabatic formulation and control simulations," Annales Geophysicae 16(1), 90-109 (1998).

[13] Masciadri, E., Lascaux, F., Turchi, A., and Fini, L., "Optical turbulence forecast: ready for an operational application," Mon. Not. R. Astron. Soc. 466, 520-539 (2017).

[14] Masciadri, E., Lascaux, F., and Fini, L., "MOSE: operational forecast of the optical turbulence and atmospheric parameters at European Southern Observatory ground-based sites - I. Overview and vertical stratification of atmospheric parameters at 0-20 km," Mon. Not. R. Astron. Soc. 436, 1968-1985 (2013).

[15] Villecroze, R., Fusco, T., Bacon, R., and Madec, P.-Y., "Psf reconstruction for muse in wide field mode," in [Adaptive Optics Systems III], Proc.SPIE 8447, 8447 - 8447 - 15 (2012).

[16] Martin, O. A., Correia, C. M., Gendron, E., Rousset, G., Gratadour, D., Vidal, F., Morris, T. J., Basden, A. G., Myers, R. M., Neichel, B., and Fusco, T., "PSF reconstruction validated using on-sky CANARY data in MOAO mode," in [Adaptive Optics Systems V], SPIE Conference Series 9909, 99091Q (July 2016).

[17] Guesalaga, A., Neichel, B., Correia, C. M., Butterley, T., Osborn, J., Masciadri, E., Fusco, T., and Sauvage, J.-F., "Online estimation of the wavefront outer scale profile from adaptive optics telemetry," Mon. Not. R. Astron. Soc. 465, 1984-1994 (Feb. 2017).

[18] Cortés, A., Neichel, B., Guesalaga, A., Osborn, J., Rigaut, F., and Guzman, D., "Atmospheric turbulence profiling using multiple laser star wavefront sensors," Mon. Not. R. Astron. Soc. 427, 2089-2099 (2012). 
[19] La Penna, P., Aller Carpentier, E., Argomedo, J., Arsenault, R., Conzelmann, R. D., Delabre, B., Donaldson, R., Gago, F., Gutierrez-Cheetam, P., Hubin, N., Jolley, P., Kiekebusch, M., Kirchbauer, J. P., Klein, B., Kolb, J., Kuntschner, H., Le Louarn, M., Lizon, J.-L., Madec, P.-Y., Manescau, A., Mehrgan, L., Oberti, S., Quentin, J., Sedghi, B., Ströbele, S., Suárez Valles, M., Soenke, C., Tordo, S., and Vernet, J., "AOF: standalone test results of GALACSI," in [Adaptive Optics Systems V], Society of Photo-Optical Instrumentation Engineers (SPIE) Conference Series 9909, 99092Z (July 2016).

[20] Ono, Y., Correia, C., Beltramo-Martin, O., Neichel, B., Fusco, T., Sauvage, J.-F., Guesalaga, A., Bechet, C., and Ayancán, B., "Turbulence profiling simulation for tomographic ao systems in elts," in [this conference], (2017).

[21] Conan, R. and Correia, C., "Object-oriented matlab adaptive optics toolbox," Proc.SPIE 9148, 9148 $9148-17$ (2014). 